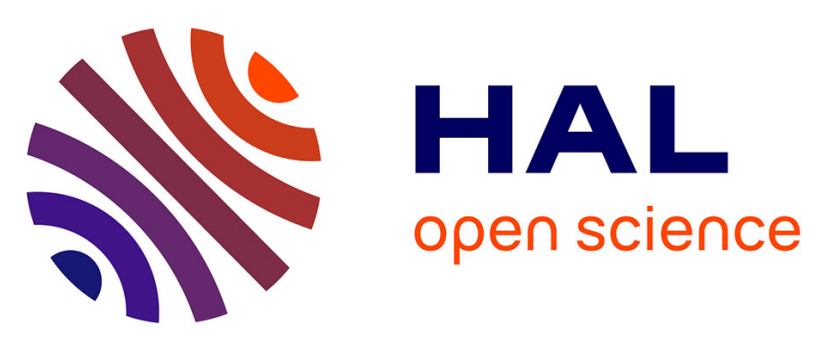

\title{
An Exact Graph Edit Distance Algorithm for Solving Pattern Recognition Problems
}

Zeina Abu-Aisheh, Romain Raveaux, Jean-Yves Ramel, Patrick Martineau

\section{To cite this version:}

Zeina Abu-Aisheh, Romain Raveaux, Jean-Yves Ramel, Patrick Martineau. An Exact Graph Edit Distance Algorithm for Solving Pattern Recognition Problems. 4th International Conference on Pattern Recognition Applications and Methods 2015, Jan 2015, Lisbon, Portugal. 10.5220/0005209202710278. hal-01168816

\section{HAL Id: hal-01168816 https://hal.science/hal-01168816}

Submitted on 26 Jun 2015

HAL is a multi-disciplinary open access archive for the deposit and dissemination of scientific research documents, whether they are published or not. The documents may come from teaching and research institutions in France or abroad, or from public or private research centers.
L'archive ouverte pluridisciplinaire HAL, est destinée au dépôt et à la diffusion de documents scientifiques de niveau recherche, publiés ou non, émanant des établissements d'enseignement et de recherche français ou étrangers, des laboratoires publics ou privés. 


\title{
An Exact Graph Edit Distance Algorithm for Solving Pattern Recognition Problems
}

\author{
Zeina Abu-Aisheh ${ }^{1}$, Romain Raveaux ${ }^{1}$, Jean-Yves Ramel ${ }^{1}$ and Patrick Martineau ${ }^{1}$ \\ ${ }^{1}$ Laboratoire d'Informatique (LI), Université François Rabelais, 37200, Tours, France \\ \{f_author,s_author\}@univ-tours.fr
}

Keywords: Graph Matching, Graph Edit Distance, Pattern Recognition, Classification.

Abstract: $\quad$ Graph edit distance is an error tolerant matching technique emerged as a powerful and flexible graph matching paradigm that can be used to address different tasks in pattern recognition, machine learning and data mining; it represents the minimum-cost sequence of basic edit operations to transform one graph into another by means of insertion, deletion and substitution of vertices and/or edges. A widely used method for exact graph edit distance computation is based on the $\mathrm{A}^{*}$ algorithm. To overcome its high memory load while traversing the search tree for storing pending solutions to be explored, we propose a depth-first graph edit distance algorithm which requires less memory and searching time. An evaluation of all possible solutions is performed without explicitly enumerating them all. Candidates are discarded using an upper and lower bounds strategy. A solid experimental study is proposed; experiments on a publicly available database empirically demonstrated that our approach is better than the $\mathrm{A}^{*}$ graph edit distance computation in terms of speed, accuracy and classification rate.

\section{INTRODUCTION}

The comparison between two objects is a crucial operation in Pattern Recognition (PR). Representing objects by graphs turns the problem of object comparison into a graph matching one where an evaluation of structural and attributed similarity of two graphs have to be found (Vento, 2015). The similarity evaluation, called matching, is based on mapping similar vertices and similar edges of the two involved graphs.

The matching problems are all NP-complete except for graph isomorphism, for which it has not yet been demonstrated if it belongs to $N P$ or not (Vento, 2015). The graph matching methods can be divided into two broad categories: exact graph matching and error-tolerant graph matching. Exact graph matching addresses the problem of detecting identical (sub)structures of two graphs $g_{1}$ and $g_{2}$ and their corresponding attributes. This category assumes the existence of only noise-free objects while in reality objects are usually affected by noise and distortion. Consequently, researchers in the PR domain often shed light on the other category, i.e., inexact graph matching, for which error-tolerance can be easily integrated into the graph matching process.

In the context of attributed graphs, the problem of error tolerant graph matching presents a higher complexity than exact graph matching as it takes distortion and noise into account during the matching process. Indeed, the exact algorithms dedicated to solving error-tolerant graph matching are computationally complex (Vento, 2015); and (M. Neuhaus and Bunke., 2006)). Consequently, lots of works have been employed to approximately solve the errortolerant graph matching problem. Such methods are often called heuristics or approximate methods. Approximate methods for the error-tolerant graph matching problem have been investigated based on genetic algorithm (Cross et al., 1997), probabilistic relaxation (Christmas et al., 1995), EM algorithm (Andrew D. J. Cross, 1998); (Finch et al., 1998) and neural networks (Kuner and Ueberreiter, 1988). The aforementioned techniques are expected to present a polynomial run-time. However, they cannot ensure the quality of their solutions and are likely to output suboptimal solutions.

Another error tolerant matching approach is achieved by a set of graph edit operations (e.g., node insertion, node deletion, etc.). The cheapest sequence of operations needed to transform one of the two graphs into another is computed. This approach is referred to in the literature as graph edit distance. In this paper, we propose a novel graph edit distance algorithm for PR problems. Our contribution is two- 
fold: a depth-first graph edit distance algorithm and the choice of meaningful optimization. This proposed algorithm is supported by a solid experimental study.

The rest of the paper is organized as follows. Section 2 is devoted to the presentation of some notations and the state-of-the-art approaches for graph edit distance. Section 3 describes the phases of our approach including a new tree search exploration and a pruning strategy for exploring less number of nodes. Section 4 defines the protocol for our extensive experiments. Section 5 presents the obtained results and provides some key remarks. Finally, conclusions are drawn and future perspectives are discussed in Section 6.

\section{RELATED WORKS}

In this section we first define our basic notations and then introduce graph edit distance and its computation.

Definition 1. (Attributed Graph)

An attributed graph (AG) is represented by a fourtuple, $\mathrm{AG}=(V, E, \mu, \zeta)$, such that:

- $V$ is a set of vertices.

- $E$ is a set of edges such as $E \subseteq V \times V$.

- $\mu: V \rightarrow L_{V}$ is a vertex labeling function which associates label $l_{V}$ to vertex $v_{i}$.

- $\zeta: E \rightarrow L_{E}$ is an edge labeling function which associates label $l_{E}$ to edge $e_{i}$.

- $L_{V}$ and $L_{E}$ are vertex and edge attributes sets, respectively. These attributes can be given by a set of integers $L=\{1 ; 2 ; 3\}$, a vector space $L=\mathbb{R}^{N}$ and/or a finite set of symbolic attributes $L=\{x ; y ; z\}$, these sets can differ in their dimensions.

Definition 1 allows to handle arbitrarily structured graphs with unconstrained labeling functions. $L_{V}$ and $L_{E}$ can be represented by numeric (e.g., 2D/3D points and the distances between them) and/or symbolic attributes (e.g., object $O_{i}$ is on top of object $O_{j}$ ).

\subsection{Graph Edit Distance}

Graph edit distance (GED) is a graph matching approach whose concept was first reported in (Sanfeliu and Fu, 1983). The basic idea of GED is to find the best set of transformations that can transform graph $g_{1}$ into graph $g_{2}$ by means of edit operations on graph $g_{1}$. The allowed operations are inserting, deleting and/or substituting vertices and their corresponding edges.

Definition 2. (Graph Edit Distance)

Let $g_{1}=\left(V_{1}, E_{1}, \mu_{1}, \zeta_{1}\right)$ and $g_{2}=\left(V_{2}, E_{2}, \mu_{2}, \zeta_{2}\right)$ be two graphs, the graph edit distance between $g_{1}$ and $g_{2}$ is defined as:

$$
G E D\left(g_{1}, g_{2}\right)=\min _{e_{1}, \cdots, e_{k} \in \gamma\left(g_{1}, g_{2}\right)} \sum_{i=1}^{k} c\left(e_{i}\right)
$$

Where $c$ denotes the cost function measuring the strength $c\left(e_{i}\right)$ of an edit operation $e_{i}$ and $\gamma\left(g_{1}, g_{2}\right)$ denotes the set of edit paths transforming $g_{1}$ into $g_{2}$.

A standard set of edit operations is given by insertions, deletions and substitutions of both vertices and edges. We denote the substitution of two vertices $u$ and $v$ by $(u \rightarrow v)$, the deletion of node $u$ by $(u \rightarrow \varepsilon)$ and the insertion of node $v$ by $(\varepsilon \rightarrow v)$. For edges $(e . g$., $w$ and $z$ ), we use the same notations as vertices. An example of an edit path between two graphs $g_{1}$ and $g_{2}$ is shown in Figure 1, the following operations have been applied in order to transform $g_{1}$ into $g_{2}$ : three edge deletions, one node deletion, one node insertion, one edge insertions and three node substitutions.

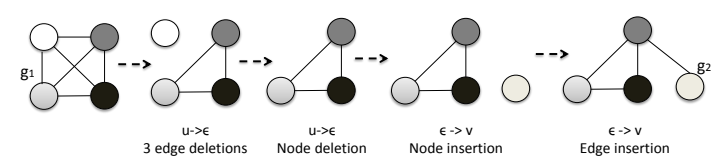

Figure 1: Transforming $g_{1}$ into $g_{2}$ by means of edit operations. Note that vertices attributes are represented in different gray scales.

Many fast heuristic methods have been proposed in the literature such as (Christmas et al., 1995); (Zeng et al., 2009); (Fankhauser et al., 2012); and (Andreas Fischer, 2013). However, these heuristic algorithms can only find unbounded suboptimal values. On the other hand, only few exact approaches have been proposed to postpone the graph size restriction (Tsai and Fu, 1979); (Justice and Hero, 2006); and (Riesen et al., 2007). Lots of exact branch and bound graph matching algorithms have been proposed in the literature. However, to the best knowledge of the authors, these works have not addressed the GED problem and cannot be easily extended to solve such a problem. For instance, a branch and bound algorithm dedicated to solving GED was proposed in (Tsai and Fu, 1979) but it was restricted to graphs that are structurally isomorphic. Afterwards, this work has been extended in (Tsai and Fu, 1983) that has taken into account insertion and deletion of nodes and edges. However, the proposed algorithm was devoted to error-correcting subgraph isomorphism.

\subsection{Exact Graph Edit Distance Computation}

A widely used method for exact GED computation is based on the $A^{*}$ algorithm (Riesen et al., 2007), 
this algorithm, referred to as $A * G E D$, is considered as a foundation work for solving GED. $A^{*} G E D$ explores the space of all possible mappings between two graphs by means of an ordered tree. Such a search tree is constructed dynamically at run time by iteratively creating successor nodes linked by edges to the currently considered node in the search tree.

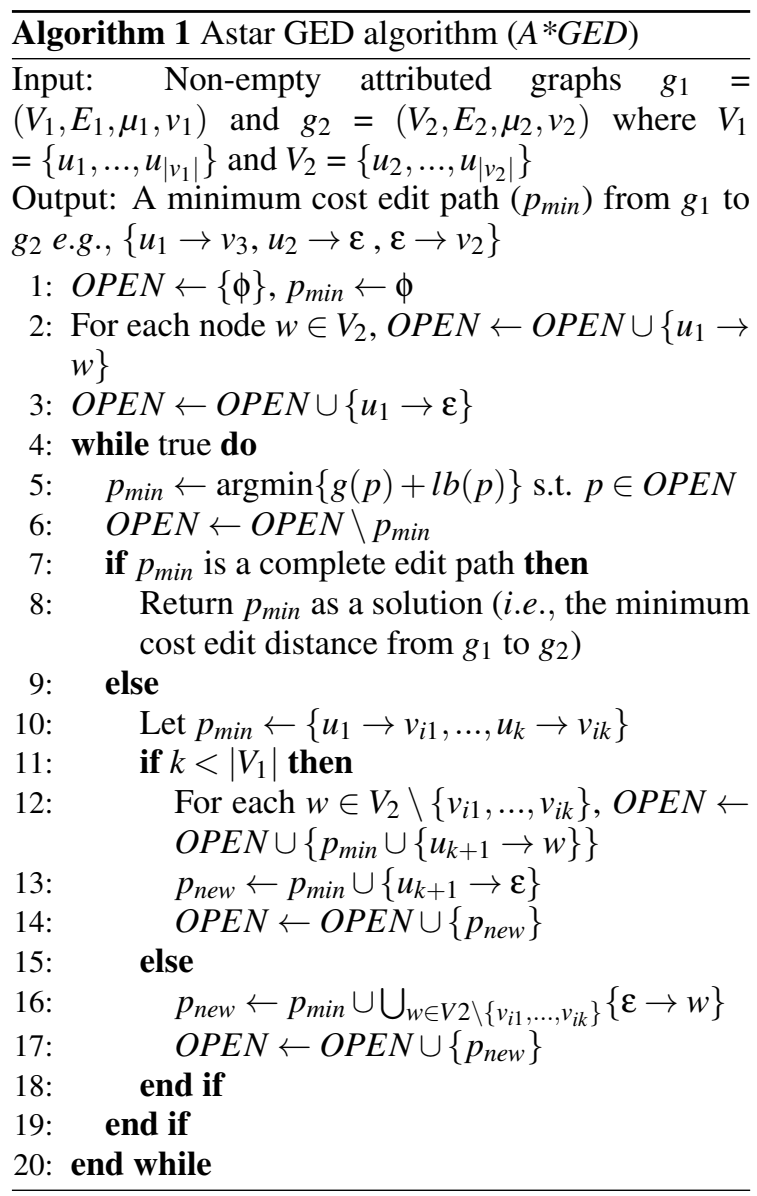

Algorithm 1 depicts the $A * G E D$ computation. In order to determine the node which is used for further expansion of the actual mapping in the next iteration, a heuristic function added to the actual partial path cost is usually used. Formally, for a node $p$ in the search tree, $g(p)$ represents the cost of the partial edit path accumulated so far, $l b(p)$ denotes the estimated costs from $p$ to a leaf node, $l b(p)$ must not underestimate the remaining cost in order to guarantee the optimality of the final solution. Also, it should be done in a faster way than the exact computation and return a good approximation of the true future cost. The sum $g(p)+l b(p)$ depicts the total cost assigned to an open node in the search tree. Obviously, the partial edit path $p$ that minimizes $g(p)+l b(p)$ is chosen next for further expansion. Note that the smaller the differ- ence between $l b$ and the real future cost, the fewer the expanded nodes. The choice of $l b$ is a crucial parameter and many lower bounds have been proposed in the literature. To the best of the authors' knowledge, the best lower bound has been presented in (Riesen and Bunke, 2009). In $A^{*} G E D, l b(p)$ is computed using an assignment algorithm on unmapped vertices and edges yet to estimate the future costs. This is performed by an assignment algorithm (Riesen and Bunke, 2009) whose complexity is $O\left(\max \left\{n_{1}, n_{2}\right\}^{3}\right)$. The estimated cost certainly constitutes a lower bound of the optimal cost as this bound represents an invalid way to edit the remaining part of $g_{1}$ into the remaining part of $g_{2}$.

\subsection{Deadlocks to be Released}

$A^{*} G E D$ is a best-first search algorithm and so the list of candidate solutions, called OPEN, grows quickly. Such a fact leads to high memory consumption and thus is considered as a bottleneck of $A^{*} G E D$. In this paper, we outperform $A * G E D$ by getting rid of high memory consumption and the re-computation of vertices and nodes matching costs. We propose a novel algorithm that reduces the used memory space using a different exploration strategy (i.e., depth-first instead of best-first). This approach also reduces the computation time as the unfruitful nodes are pruned by the lower and upper bounds strategy. A preprocessing strategy is included. First, edges and vertices costs matrices are constructed to get rid of re-computation when exploring nodes in the search tree. Second, the list $V_{1}$ is sorted to speed up the search for the best edit path to be explored.

\section{OUR PROPOSAL}

In order to get rid of the high memory consumption and to converge faster to the optimal solution, we propose a depth-first GED ( $D F-G E D)$. The elements of the algorithm are described in Sections 3.1 to 3.6. Moreover, a pseudo-code is presented in Section 3.7.

\subsection{Structure of Search-Tree Nodes}

From now on, we will refer to the search-subtree rooted in node $p$ as Partial Edit Path $(p)$. Figure 2 illustrates an example of a partial edit path.

Each $p$ is then identified by the following elements:

- matched-vertices $(p)$ and matched-edges $(p)$ : the elements contained in these sets are vertices and edges that have been matched so far in both $g_{1}$ 


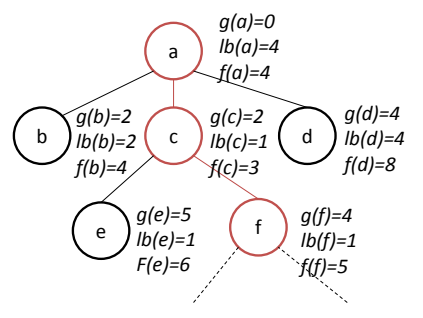

Figure 2: An example of a partial edit path $p$ whose explored nodes so far are $a, c$ and $f . f(*)=g(*)+l b(*)$.

and $g_{2}$. These sets can contain substitution $(u \rightarrow$ $v)$, deletion $(u \rightarrow \varepsilon)$ and/or insertion $(\varepsilon \rightarrow v)$ of vertices and edges, correspondingly.

- pending-vertices $_{i}(p)$ and pending-edges $(p)$ : these sets represent vertices and edges of both $g_{1}$ and $g_{2}$ (i.e., $V_{1}, V_{2}, E_{1}$ and $E_{2}$ ) that are not substituted, deleted or inserted yet where pending-vertices $_{1}(p)$ and pending-vertices $s_{2}(p)$ represent pending $V_{1}$ and pending $V_{2}$, respectively whereas pending-edges $s_{1}(p)$ and pending$\operatorname{edges}_{2}(p)$ represent pending $E_{1}$ and pending $E_{2}$, respectively.

- $\operatorname{parent}(p)$ : the parent of $p$.

- children $(p)$ : for any node $p$, the exploration is achieved by choosing the next most promising vertex $u_{i}$ of pending-vertices $s_{1}(p)$ and matching it with all the elements of pending-vertices $s_{2}(p)$ in addition to the deletion of this node (i.e., $u_{i} \rightarrow \varepsilon$ ). All these matchings are referred to as children $(p)$.

- $l b(p)$ : the lower bound, $l b$, of the estimated future cost from node $p$ does not underestimate the complete solution. The calculation of the lower bound is described in Section 3.5.

- $g(p)$ : the cost of matched-vertices $(p)$ and matched-edges $(p)$. Both $l b$ and $g$ depend on the attributes as well as the structure of the involved sub-trees. The cost functions involved with each PR dataset permit to calculate insertions, deletions and substitutions of vertices and/or edges.

\subsection{Preprocessing}

Preprocessing is applied before the branch and bound procedure starts in order to speed up the tree search exploration. First, vertices and edges cost matrices are constructed. Second, vertices-sorting is conducted.

\subsubsection{Cost Matrices}

The vertices and edges cost matrices $\left(C_{v}\right.$ and $\left.C_{e}\right)$ are constructed, respectively. This step aims at speeding up branch and bound by getting rid of re-calculating the assigned costs when matching vertices and edges of $g_{1}$ and $g_{2}$.

Let $g_{1}=\left(V_{1}, E_{1}, \mu_{1}, \xi_{1}\right)$ and $g_{2}=\left(V_{2}, E_{2}, \mu_{2}, \xi_{2}\right)$ be two graphs with $V_{1}=\left(u_{1}, \ldots, u_{n}\right)$ and $V 2=$ $\left(v_{1}, \ldots, v_{m}\right)$. A vertices cost matrix $C_{v}$, whose dimension is $(n+2) \mathrm{X}(m+2)$, is constructed as follows:

$$
C_{v}=\mid \begin{array}{cccc||cc}
c_{1,1} & \ldots & \ldots & c_{1, m} & c_{1 \leftarrow \varepsilon} & c_{1 \rightarrow \varepsilon} \\
\ldots & \ldots & \ldots & \ldots & \ldots & \ldots \\
\ldots & \ldots & \ldots & \ldots & \ldots & \ldots \\
c_{n, 1} & \ldots & \ldots & c_{n, m} & c_{n \leftarrow \varepsilon} & c_{n \rightarrow \varepsilon} \\
\hline \hline c_{\varepsilon \rightarrow 1} & \ldots & \ldots & c_{\varepsilon \rightarrow m} & \infty & \infty \\
c_{\varepsilon \leftarrow 1} & \ldots & \ldots & c_{\varepsilon \leftarrow m} & \infty & \infty
\end{array}
$$

where $n$ is the number of vertices of $g_{1}$ and $m$ is the number of vertices of $g_{2}$.

Each element $c_{i, j}$ in the matrix $C_{v}$ corresponds to the cost of assigning the $i^{t h}$ vertex of the graph $g_{1}$ to the $j^{t h}$ vertex of the graph $g_{2}$. The left upper corner of the matrix contains all possible node substitutions while the right upper corner represents the cost of all possible vertices insertions and deletions of vertices of $g_{1}$, respectively. The left bottom corner contains all possible vertices insertions and deletions of vertices of $g_{2}$, respectively whereas the bottom right corner elements cost is set to infinity which concerns the substitution of $\varepsilon-\varepsilon$.

$C_{e}$ contains all the possible substitutions, deletions and insertions of edges of $g_{1}$ and $g_{2} . C_{e}$ is constructed in the very same way as $C_{v}$.

\subsubsection{Vertices-Sorting Strategy}

As $G E D$ aims at transforming $g_{1}$ into $g_{2}$ so it is important to sort $V_{1}$ in order to start with the most promising vertices that will speed up the exploration of the search tree while searching for the optimal solution. The aforementioned $C_{v}$ is used as an input of the vertices-sorting phase. To sort $V_{1}$, Munkres' algorithm is applied. From now on, we will refer to the set of sorted vertices as sorted- $V_{1}$.

\subsection{Branching Strategy}

A systematic evaluation of all possible solutions is performed without explicitly evaluating all of them. The solution space is organized as an ordered tree which is explored in a depth-first way. In depth-first search, each node is visited just before its children. In other words, when traversing the search tree, one should travel as deep as possible from node $i$ to node $j$ before backtracking.

The root $r$ of the search-tree is the node with matched-vertices $(r)=\{\phi\}$, matched-edges $(r)=\{\phi\}$, 
pending-vertices $_{1,2}(r)=V_{1} \cup V_{2}$, pending-edges pen, $_{1,2}(r)$ $=E_{1} \cup E_{2}, g(r)=\infty$ and $l b(r)=\infty$. Initially $r$ is the only node in the set OPEN i.e., the set of the edit paths, found so far. The exploration starts with the first most promising vertex $u_{1}$ in sorted $-V_{1}$ in order to generate the root's children children $(r)$. Then, chil$\operatorname{dren}(r)$ is added to OPEN. Consequently, a minimum edit path $\left(p_{\min }\right)$ is chosen to be explored by selecting the minimum cost node (i.e., $\min (g(p)+l b(p)))$ among children $(r)$ and so on. We backtrack to continue the search for a good edit path by revoking $p_{\text {min }}$ (if $p_{\text {min }}$ equals $\phi$ ) and trying out the next child in the set of children( $r$ ) and so on.

\subsection{Reduction Strategy}

As in $A^{*} G E D$, pruning, or bounding, is achieved thanks to $l b(p), g(p)$ and a global upper bound $U B$ obtained at node leaves. Formally, for a node $p$ in the search tree, the sum $g(p)+l b(p)$ is taken into account and compared with $U B$. That is, if $g(p)+l b(p)$ is less than $U B$ then $p$ can be explored. Otherwise, the encountered $p$ will be pruned from OPEN and a backtracking is done looking for the next promising node and so on until finding the best $U B$ that represents the optimal solution of $D F-G E D$. This algorithms differs from $A^{*} G E D$ as at any time $t$, in the worst case, OPEN contains approximately $\left|V_{1}\right| .\left|V_{2}\right|$ elements and hence the memory consumption is not exhausted.

\subsection{Lower Bound}

The lower bound $l b(p)$, adapted to $D F-G E D$, is the one used in section $A * G E D$, see Section 2.2.

\subsection{Upper Bound}

The very first upper bound $(U B)$ is computed by Munkres' algorithm as it provides reasonable results, see (Riesen and Bunke, 2009) for more details. Afterwards and while traversing the search tree, $U B$ is replaced by the best $U B$ found so far (i.e., a complete path whose cost is less than the current $U B$ ). After finishing the traversal of the search tree (i.e., when $p_{\text {min }}$ equals $\phi$ and its parent is $r$ ), the best $U B$ is outputted as an optimal solution of $D F-G E D$. Encountering upper bounds when performing a depth-first traversal efficiently prunes the search space and thus helps at finding the optimal solution faster than $A^{*} G E D$.

\subsection{Pseudo Code}

As depicted in Algorithm 2, DF-GED starts by a preprocessing step (line 2), then an upper bound $U B$ is calculated by Munkres' algorithm (line 3). The traversal of the search tree starts by selecting a first vertex $u_{1} \in$ sorted $-V_{1}$ where $u_{1}$ substituted with all vertex $w$ in graph $g_{2}$ as well as the deletion case $\left(u_{1} \rightarrow \varepsilon\right)$ are inserted into OPEN (lines 4 and 5). A branching step is performed in line 8 where the best child $p_{\text {min }}$ is selected, the backtracking is done when there is no more children to explore in the selected branch and the parent node (parent $t_{\text {tmp }}$ ) is not the root (lines 9 to 12 ). $p_{\min }$ is explored by substituting the next promising node $u_{k+1}$ with pendingvertices $_{2}\left(p_{\text {min }}\right)$ and also deleting $u_{k+1}$, respectively (lines 18 to 24$)$. Similar to $A^{*} G E D$, if pendingvertices ${ }_{1}\left(p_{\text {min }}\right)$ equals $\phi$, pending-vertices ${ }_{2}\left(p_{\text {min }}\right)$ will be inserted (line 26). $U B$ and Best-Edit-Path are updated whenever a better $U B$ is encountered (line 28). Each time the next parent to be explored will be replaced by $p_{\min }$ (line32). This algorithm guarantees to find the optimal solution of $G E D\left(g_{1}, g_{2}\right)$. Note that edges operations are taken into account in the matching process when substituting, deleting or inserting their corresponding vertices.

\section{EXPERIMENTS}

\subsection{Environment}

Evaluations are conducted on a 4-core Intel i7 processor $3.07 \mathrm{GHz}$ and $8 \mathrm{~GB}$ of memory.

\subsection{Compared Methods}

We compare $A^{*} G E D$ with $D F-G E D$. Both methods return a node to node matching.

\subsection{Database in Use}

To the best of our knowledge, few publicly available graphs databases are dedicated to graph matching tasks. However, most of these datasets consist of synthetic graphs that are not representative of PR problems concerning graph matching under noise and distortion. We shed light on the IAM graph repository which is a widely used repository dedicated to a wide spectrum of tasks in pattern recognition and machine learning (Riesen and Bunke, 2008). Moreover, it contains graphs of both symbolic and numeric attributes which is not often the case of other datasets.

The GREC database used in our experiments is a dataset in IAM which consists of a subset of the symbol database underlying the GREC2005 competition (Dosch and Valveny, 2006). The images of GREC represent symbols from architecture, electronics, and 


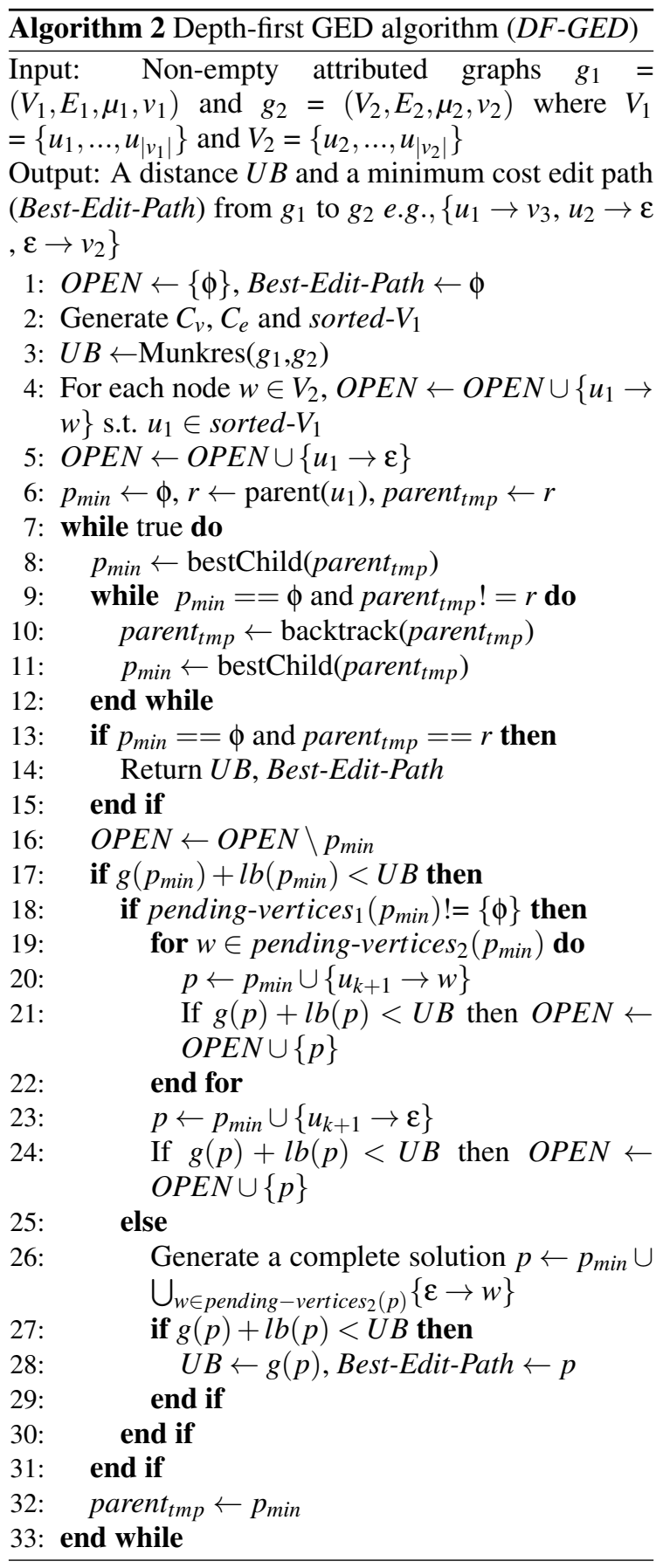

other technical fields. Distortion operators are applied to the original images in order to simulate handwritten symbols. To this end, the primitive lines of the symbols are divided into subparts. The ending points of these subparts are then randomly shifted within a certain distance, maintaining connectivity. Each node represents a subpart of a line and is attributed with its relative length (ratio of the length of the actual line to the length of the longest line in the symbol). Connection points of lines are represented by edges attributed with the angle between the corresponding lines. The dataset consists of 6 classes and 30 instances per class. This dataset is useful as both vertices and edges are labeled with numeric attributes. In addition, it holds graphs whose sizes vary from 5 to 25 vertices.

Cost Function: The vertices of graphs from GREC dataset are labeled with $(\mathrm{x}, \mathrm{y})$ coordinates and a type (ending point, corner, intersection or circle). The same accounts for the edges where two types (line, arc) are employed. The Euclidean cost model is adopted accordingly. That is, for node substitutions the type of the involved vertices is compared first. For identically typed vertices, the Euclidean distance is used as node substitution cost. In case of nonidentical types on the vertices, the substitution cost is set to $2 \tau_{\text {node }}$, which reflects the intuition that vertices with different type label cannot be substituted but have to be deleted and inserted, respectively. For edge substitutions, the dissimilarity of two types is measured with a Dirac function returning 0 if the two types are equal, and $2 \tau_{e d g e}$ otherwise. Both $\tau_{\text {node }}$ and $\tau_{e d g e}$ are non-negative parameters. To control whether the edit operation cost on the vertices or on the edges is more important another parameter $\alpha$ is integrated. In our experiments we have set $\tau_{\text {node }}, \tau_{\text {edge }}$ and $\alpha$ to 90, 15 and 0.5, respectively. These meta parameters' values are taken from (Riesen and Bunke, 2010). This dataset consists of 1,100 graphs where graphs are uniformly distributed between 22 symbols. The resulting dataset is split into a training and a validation set of size 286 each, and a test set of size 528 .

GREC Decomposition: We decompose the database into subsets, each of which contains graphs that have the same size. We focus on the following subsets: (GREC5, GREC10, GREC15 and GREC20) aiming at evaluating the two methods when increasing the size of the involved graphs (i.e., the number of vertices). Due to the large number of matchings considered and the exponential complexity of the tested algorithms, we select 10 graphs of the train set of each subset of GREC. The train set is representative of all graph distortions and the selection of only 10 graphs per subset ends up having 100 pairwise comparisons which is significant for such a kind of experiments. Furthermore, we add another subset GREC-mix that contains different number of vertices (i.e., 10 graphs with different number of vertices). 


\subsection{Protocol and Quality Measures}

Our Protocol is two-fold. First, calculating the distance matrix under small and big time constraints. Second, Classification test under a reasonable time constraint.

Let $\mathcal{S}$ be a graph dataset consisting of $m$ graphs, $\mathcal{S}=\left\{g_{1}, g_{2}, \ldots, g_{m}\right\}$. Let $\mathcal{P}=\{\mathrm{A} *$ GED, DF-GED $\}$ be the set the compared methods. Given a method $p \in \mathcal{P}$, we computed the square distance matrix $M^{p} \in \mathcal{M}^{m}\left(\mathbb{R}^{+}\right)$, that holds every pairwise comparison $M_{i, j}^{p}=d_{p}\left(g_{i}, g_{j}\right)$, where the distance $d_{p}\left(g_{i}, g_{j}\right)$ is the value returned by the method $p$ on the graph pair $\left(g_{i}, g_{j}\right)$ within a certain time and memory limits. Hence, $M^{\mathrm{A} * \mathrm{GED}}$ and $M^{\mathrm{DF}-\mathrm{GED}}$ denote distance matrices of $A * G E D$ and $D F-G E D$ methods, respectively.

Let $G T \in \mathcal{M}^{m}\left(\mathbb{R}^{+}\right)$be the reference matrix that holds the best found distance for each pair of graphs. We aim at comparing the errors committed by the two methods as well as their running time under a time constraint $C_{T}$ and a memory constraint $C_{M}$ when graphs' sizes increase (i.e., on GREC $k$ ) and also on GREC-mix. To this objective, we test the accuracy of $\mathcal{P}$ when $C_{T}$ is small, e.g., $C_{T}=350$ milliseconds (ms), and when $C_{T}$ is big (e.g., $C_{T}=5$ minutes). $C_{M}$ is set to $1 \mathrm{~GB}$ during all the experiments. We expect $A^{*} G E D$ to violate $C_{M}$ specially when graphs get larger.

In the following, we define the measurements used for evaluating our protocol:

Deviation: We evaluate the error committed by a method $p$ over the reference distances. To this end, we measure an indicator called deviation and defined by the following equation:

$$
\operatorname{deviation}(i, j)^{p}=\frac{\left|M_{i, j}^{p}-G T_{i, j}\right|}{G T_{i, j}}, \forall(i, j) \in \llbracket 1, m \rrbracket^{2}
$$

Where $G T_{i, j}$ is the smallest distance among all distances generated by $p$ when matching $g_{i}$ and $g_{j}$.

Running Time: We measure the running time in milliseconds for each comparison $d\left(g_{i} ; g_{j}\right)$. This value reflects the overall time for GED computation including all the inherits costs computations (i.e., $g(p) ; l b(p)$; and $U B)$.

For the classification test, we are interested in the average computation time $(t)$ which corresponds to the average time elapsed when classifying all the test graphs of GREC and the classification accuracy $(A C)$ which defines the error made when classifying the test graphs of GREC. Both measurements are achieved when $C_{T}=500 \mathrm{~ms}$ and $C_{M}=1 \mathrm{~GB}$. The classification stage is performed by a $1-\mathrm{NN}$ classifier. Each test graph $g_{t}$ is compared to the entire training set. The nearest neighbor's label is assigned to $g_{t}$.

\section{RESULTS AND DISCUSSION}

Figure 3 shows the deviation results under $350 \mathrm{~ms}$ and 5 minutes respectively. We observe that $D F-G E D$ always outperforms $A * G E D$ under the same time constraint. For all GED computations, $D F-G E D$ gives the best distance (i.e., $G T_{i, j}$ ) and so its deviation is always $0 \%$. In contrast with $D F-G E D$, the deviation of $A^{*} G E D$ decreases when $C_{T}=5$ minutes. However, when the size of graphs increase (e.g., GREC15 and GREC20), the deviation starts to converge due to memory saturation where the best recently known solution is outputted before halting.
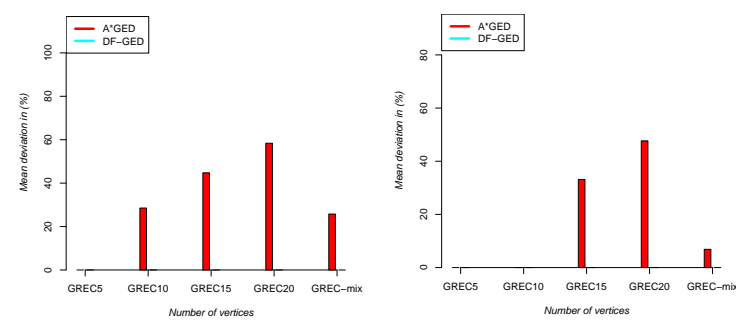

Figure 3: Deviation: Left (350 ms), Right (5 minutes).

Figure 4 demonstrates the running time of both $A^{*} G E D$ and $D F-G E D$. When $C_{T}=350 \mathrm{~ms}$ both running times are relatively equal on GREC15 and GREC20 and that is because non of them is able to find an optimal solution before exceeding $C_{T}$. When $C_{T}$ increases, $D F-G E D$ becomes faster as it explores the search tree in a depth-first way (i.e., not stopped by $C_{M}$ ) while pruning the search tree thanks to its upper and lower bounds as well as the preprocessing step, see Section 3.2. On the other hand, $A^{*} G E D$ does not continue for further exploration on GREC15 and GREC20 because of the size of the involved graphs where available memory is exhausted and so the best recently known solution is given before halting.
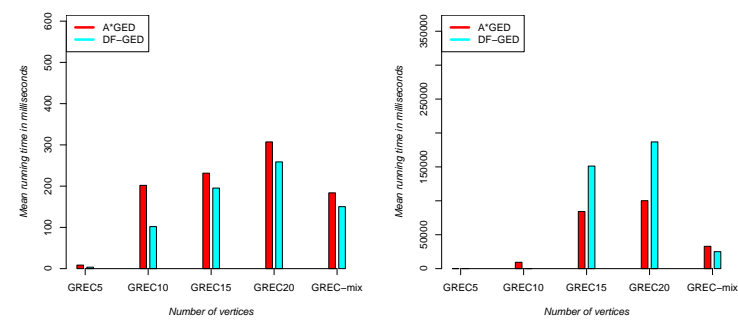

Figure 4: Running Time: Left (350 ms), Right (5 minutes).

For the classification experiment, 151008 comparisons are performed on 286 training graphs and 528 test graphs of the GREC dataset. As depicted in Table 1 , results show that the classification accuracy $A C$ of $D F-G E D$ is 2.3 times higher under the same $C_{T}$ where $C_{T}=500 \mathrm{~ms}$. Moreover, $D F-G E D$ is 1.9 times faster as 
the average time $t$ is smaller.

\begin{tabular}{|c|c|c|}
\hline Algorithms & $t$ & $A C$ \\
\hline \hline$A^{*} G E D$ & $119491.5 \mathrm{~ms}$ & $42.23 \%$ \\
\hline$D F-G E D$ & $60468.7 \mathrm{~ms}$ & $98.48 \%$ \\
\hline
\end{tabular}

Table 1: Classifying graphs of GREC $\left(C_{T}=500 \mathrm{~ms}\right)$.

\section{CONCLUSION AND PERSPECTIVES}

In the present paper, we have considered the problem of GED computation for PR. Graph edit distance is a powerful and flexible paradigm that has been used in different applications in PR. The exact algorithm, $A * G E D$, presented in the literature suffers from high memory consumption and thus is too costly to match large graphs. In this paper, we propose another exact GED algorithm, DF-GED, which is based on depth-first search. This algorithm speeds up the computations of graph edit distance thanks to its upper and lower bounds pruning strategy and its preprocessing step. Moreover, this algorithm does not exhaust memory as the number of pending edit paths that are stored in the set $O P E N$ is relatively small thanks to the depth-first search where the number of pending nodes is $|V 1| .|V 2|$ in the worst case.

In the experimental section, we have proposed to evaluate sub-optimally both exact methods: $A * G E D$ and $D F-G E D$ under some memory and time constraints. Experiments on the GREC database empirically demonstrated that $D F-G E D$ outperforms $A^{*} G E D$ in terms of precision, speed and classification rate. In future work, we aim at proposing a benchmark to measure the quality of the solutions found by approximate methods. Both exact and approximate graph edit distance computations will be evaluated on different PR databases.

\section{REFERENCES}

Andreas Fischer, Ching Y. Suen, V. F. K. R. H. B. (2013). A fast matching algorithm for graph-based handwriting recognition. GbRPR 2013, 7877:194-203.

Andrew D. J. Cross, E. R. H. (1998). Graph matching with a dual-step em algorithm. IEEE Trans. Pattern Anal. Mach. Intell., 20(11):1236-1253.

Christmas, W., Kittler, J., and Petrou, M. (1995). Structural matching in computer vision using probabilistic relaxation. Pattern Analysis and Machine Intelligence, IEEE Transactions on, 17(8):749-764.
Cross, A. D., Wilson, R. C., and Hancock, E. R. (1997). Inexact graph matching using genetic search. Pattern Recognition, 30(6):953-970.

Dosch, P. and Valveny, E. (2006). Report on the second symbol recognition contest. 3926:381-397.

Fankhauser, S., Riesen, K., Bunke, H., and Dickinson, P. J. (2012). Suboptimal graph isomorphism using bipartite matching. IJPRAI, 26(6).

Finch, A., Wilson, R., and Hancock, E. (1998). An energy function and continuous edit process for graph matching. Neural Computation, 10(7):1873-1894.

Justice, D. and Hero, A. (2006). A binary linear programming formulation of the graph edit distance. IEEE Transactions on Pattern Analysis and Machine Intelligence, 28(8):1200-1214.

Kuner, P. and Ueberreiter, B. (1988). Pattern recognition by graph matching: Combinatorial versus continuous optimization. International Journal in Pattern Recognition and Artificial Intelligence, 2(3):527-542.

M. Neuhaus, K. R. and Bunke., H. (2006). Fast suboptimal algorithms for the computation of graph edit distance. Proceedings of 11th International Workshop on Structural and Syntactic Pattern Recognition., 28:163-172.

Riesen, K. and Bunke, H. (2008). Iam graph database repository for graph based pattern recognition and machine learning. pages 287-297.

Riesen, K. and Bunke, H. (2009). Approximate graph edit distance computation by means of bipartite graph matching. Image and Vision Computing, 27(7):950 959.

Riesen, K. and Bunke, H. (2010). Graph Classification and Clustering Based on Vector Space Embedding.

Riesen, K., Fankhauser, S., and Bunke, H. (2007). Speeding up graph edit distance computation with a bipartite heuristic. In Mining and Learning with Graphs, MLG 2007, Proceedings.

Sanfeliu, A. and Fu, K. (1983). A distance measure between attributed relational graphs for pattern recognition. IEEE Transactions on Systems, Man, and Cybernetics, 13(3):353-362.

Tsai, W.-H. and Fu, K.-S. (1979). Error-correcting isomorphisms of attributed relational graphs for pattern analysis. Systems, Man and Cybernetics, IEEE Transactions on, $9(12): 757-768$.

Tsai, W.-H. and Fu, K.-S. (1983). Subgraph error-correcting isomorphisms for syntactic pattern recognition. Systems, Man and Cybernetics, IEEE Transactions on, SMC-13(1):48-62.

Vento, M. (2015). A long trip in the charming world of graphs for pattern recognition. Pattern Recognition, 48(2):291-301.

Zeng, Z., Tung, A. K. H., Wang, J., Feng, J., and Zhou, L. (2009). Comparing stars: On approximating graph edit distance. Proc. VLDB Endow., 2(1):25-36. 